\title{
Mass sensitivity of acoustic wave devices from group and phase velocity measurements
}

\author{
G. McHale, ${ }^{\text {a) }}$ F. Martin, and M. I. Newton \\ Department of Chemistry and Physics, Nottingham Trent University, Clifton Lane, \\ Nottingham NG11 8NS, United Kingdom
}

(Received 26 March 2002; accepted for publication 18 June 2002)

The effect of dispersion on acoustic wave sensors is considered. The discussion is focused upon layer guided surface acoustic waves (Love waves), which obtain their high mass sensitivity for the first Love wave mode by optimizing the guiding layer thickness, $d$, such that $d \sim \lambda_{l} / 4$; the wavelength in the layer is given by $\lambda_{l}=f / v_{l}$ where $f$ is the operating frequency and $v_{l}$ is the shear acoustic speed of the guiding layer. We show that this optimization of guiding layer thickness corresponds to strong dispersion so that the phase and group velocities can be quite different. From the definition of the phase velocity mass sensitivity, we show that it can be determined from either the slope of the curve of phase velocity with normalized guiding layer thickness, $z=d / \lambda_{l}$, or from the phase and group velocities measured for a given guiding layer thickness. Experimental data for a poly(methylmethacrylate) polymer guiding layer on $36^{\circ} X Y$ Lithium Tantalate is presented. Measurements of phase velocity and group velocity determined by a network analyzer were obtained for systematically increasing guiding layer thicknesses; a pulse transit experiment was also used to provide independent confirmation of the group velocity data. Two independent estimates of the mass sensitivity are obtained for $z=d / \lambda_{l}<0.22$ from (i) the slope of the phase velocity curve and (ii) the measurements of the group and phase velocity. These two estimates are shown to be consistent and we, therefore, conclude that it is possible to determine the mass sensitivity for a Love wave device with a given guiding layer thickness from measurements of the phase and group velocities. Moreover, we argue that the formula using group velocity to determine phase velocity mass sensitivity can be extended to a wide range of other acoustic wave sensors. In addition, we suggest that variations in the group velocity due to deposited mass may be a more sensitive parameter than variations in the phase velocity. (C) 2002 American Institute of Physics. [DOI: $10.1063 / 1.1499750$ ]

\section{INTRODUCTION}

Acoustic wave sensors are being increasingly investigated for their potential in gas and liquid phase sensor applications. ${ }^{1-4}$ Among the most mass sensitive of these sensors are acoustic waves utilizing some form of guiding. These include Love wave and surface transverse wave (STW) devices which combine a shear horizontally polarized surface acoustic wave (SAW) delay line with either a guiding layer ${ }^{5,6}$ or a surface grating structure, respectively, to slow down the wave and further confine it to the surface. ${ }^{7}$ This type of acoustic wave mode necessarily involves a system with dispersion so that the phase velocity is not equal to the group velocity. While some recognition of the difference between group and phase velocity exists in literature on acoustic plate mode sensors, ${ }^{8-10}$ relatively little discussion of this has occurred in literature on Love wave sensors. ${ }^{11,12}$ The main effect that has been accounted for is the inclusion of a factor, which is the ratio of the group to phase velocity, in the formula $\Delta f / f=\left(v_{g} / v\right)(\Delta v / v)$ relating the fractional frequency shift to the fractional change in phase speed due to mass deposition. However, this does not represent a detailed consideration of the effect of dispersion on acoustic wave

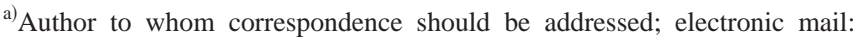
glen.mchale@ntu.ac.uk
}

sensors and how it influences the mass sensitivity. Moreover, there are recent reports in the literature of the use of pulse transit type experiments ${ }^{13,14}$ and it is therefore timely to seek a better understanding of the effects of dispersion.

In this article, we discuss the relationship between the phase velocity and the group velocity and the mass sensitivity of acoustic wave sensors that use acoustic modes with dispersion. To show a specific application of the concepts being developed, we focus the discussion upon Love wavetype sensors, but we emphasize that the concepts themselves are valid for other types of acoustic wave sensors showing strong dispersion. In the theoretical part of the article, we illustrate the angular frequency-wave vector dispersion curve for the first three Love wave modes and from this compare the group and phase velocities for Love waves. A relationship between the phase velocity mass sensitivity and the slope of the dispersion curve is then used to derive a simple formula relating measurements of group and phase velocity to the phase velocity mass sensitivity. The utility of such a formula is that it enables the mass sensitivity of a device to be assessed experimentally without the need to deposit additional material. We also argue that the formula can be extended to other types of acoustic wave sensors. In addition, we suggest that the group velocity may be a more sensitive parameter indicating deposited mass than the phase velocity. In the second part of the article, we present experimental 


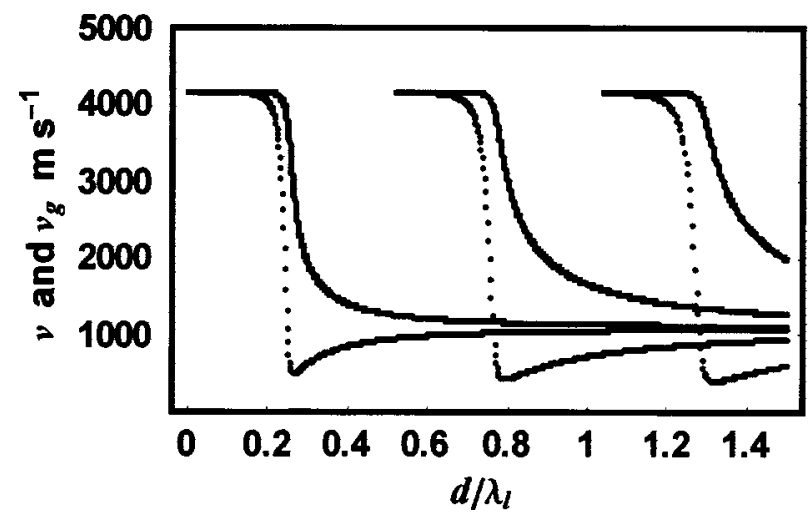

FIG. 1. Calculated Love wave (first, second, and third modes) phase speed curves (solid lines) for an infinitely thick substrate with shear speed and density of $v_{s}=4160 \mathrm{~m} \mathrm{~s}^{-1}$ and $\rho_{s}=7456 \mathrm{~kg} \mathrm{~m}^{-3}$ covered by a guiding layer with shear speed and densities of $v_{l}=1100 \mathrm{~m} \mathrm{~s}^{-1}$ and $\rho_{l}=1000 \mathrm{~kg} \mathrm{~m}^{-3}$. The dotted curves shows the corresponding group speeds calculated using a $0.25 \mu \mathrm{m}$ thick guiding layer.

results for the change of the phase velocity of the first Love wave mode on $\mathrm{LiTaO}_{3}$ with the change in thickness of a poly(methylmethacrylate) (PMMA) guiding layer. We then determine the phase velocity mass sensitivity at a range of guiding layer thicknesses from the variation of the phase velocity with guiding layer thickness. We also use direct measurements of the group velocity, measured using two independent experimental configurations, and the phase velocity to provide a second complementary estimate of the phase velocity mass sensitivity. The comparison of the two methods of obtaining the mass sensitivity suggests that measurements of group and phase velocity provide a simple method of estimating the phase velocity mass sensitivity of a device. Finally, we show that the change in group velocity with deposited mass is a highly sensitive parameter that may have potential in sensors.

\section{THEORETICAL DISCUSSION}

\section{A. Phase and group velocity}

In a Love wave, the higher mass sensitivity can be understood as a consequence of the change of the phase speed, $v$, as a function of the normalized guiding layer thickness, $z=d / \lambda_{l}$ where $d$ is the guiding layer thickness and $\lambda_{l}$ is the characteristic shear acoustic wavelength of the guiding layer at the operating frequency, $f$ (i.e., $\lambda_{l}=v_{l} / f$, where $v_{l}$ is the shear acoustic speed of the layer). ${ }^{15,16}$. For small guiding layer thicknesses, the speed of the first Love wave is close to the shear acoustic speed of the substrate, $v_{s}$, while for large $z$, the speed becomes close to that of the shear acoustic speed of the guiding layer, $v_{l}$. The solid curves in Fig. 1 show a calculation of the Love wave phase speed, $v$, for the first three Love wave modes supported by an infinitely thick isotropic substrate coated with a waveguide layer. The substrate shear speed and densities are $v_{s}=4160 \mathrm{~ms}^{-1}$ and $\rho_{s}=7456$ $\mathrm{kg} \mathrm{m}^{-3}$ and the layer shear speed and densities are $v_{l}=1100$ $\mathrm{ms}^{-1}$ and $\rho_{l}=1000 \mathrm{~kg} \mathrm{~m}^{-3}$. In a Love wave sensor, the transition of the Love wave speed between the two limiting cases of $v_{s}$ and $v_{l}$ is rapid with guiding layer thickness, so that operating the Love wave device at the point of steepest change in the phase speed gives high mass sensitivity (see $z \sim 0.25$ for the first Love wave mode in Fig. 1). Depositing a thin mass layer appears similar to increasing the guiding layer thickness and so causes large changes in the phase speed from that at the operating point. However, this type of relationship between phase speed and normalized guiding layer thickness means that the system can have strong dispersion so that the phase and group velocities will not always be the same. The phase velocity, $v$, is defined using the frequency and wavelength as $v=f \lambda$, or equivalently as $v$ $=\omega / k$ when using the angular frequency $\omega=2 \pi f$ and wave vector $k=2 \pi / \lambda$. The group velocity, $v_{g}=d \omega / d k$, is the slope of the $(\omega, k)$ dispersion curve. This dispersion has a significant effect when pulses are used rather than a continuous wave with a single frequency.

Physically, the phase velocity is the speed at which a particular sinusoidal wave travels. When a pulse is made by combining a number of sinusoidal waves, each wave will travel with its own phase speed. In the dispersionless case, these phase speeds are all constant, independent of frequency, and the pulse therefore travels at a constant speed without altering its shape; this is the case for the Love wave when $z$ is either small or large. However, when dispersion occurs, the pulse will travel at a characteristic speed of its own and the pulse will spread out as it travels; this is the case for the Love wave when $z$ is intermediate in value and this corresponds to the operating region giving maximum phase sensitivity. The group velocity is the velocity at which the energy in the pulse is transmitted. For a given guiding layer thickness, the frequency components in a pulse each have a slightly different value of $z$ and this gives rise to different phase speeds. These speeds do not have a significant effect on a pulse unless the slope of the phase speed curve with $z$ is large, but for a Love wave sensor this itself is the requirement for high mass sensitivity. The fact that a pulse possesses a small range of frequencies and each frequency component therefore sees a slightly different effective thickness $z=d f / v_{l}$ of guiding layer means the pulse effectively samples the local slope of the curve of phase speed with $z$. Since this slope determines the mass sensitivity, it is possible to anticipate that mass sensitivity could be probed by measurements of the group velocity.

\section{B. Dispersion curve}

Considering the solid curves in Fig. 1, it is apparent that the group and phase velocities of the first Love wave mode are identical for both small $z$ and large $z$ because changing the value of $z$ by altering the frequency does not cause large changes in the phase velocity. For low $z$, the phase and group velocities will both be close to the substrate shear speed, $v_{s}$, while for large $z$, the phase and group velocities will both be close to the guiding layer shear speed, $v_{l}$. The $(\omega, k)$ dispersion curve can be calculated from the $(v, z)$ curve using $\omega$ $=2 \pi z v_{l} / d$ and $k=\omega / v=2 \pi z v_{l} /(v d)$ provided the ratio $v_{l} / d$ of the layer shear speed to the layer thickness is known. The dotted curves in Fig. 1, given the group velocities, are calculated for a specific layer speed of $v_{l}=1100 \mathrm{~ms}^{-1}$ and so the ratio $v_{l} / d$ corresponds to a specific choice of the layer 


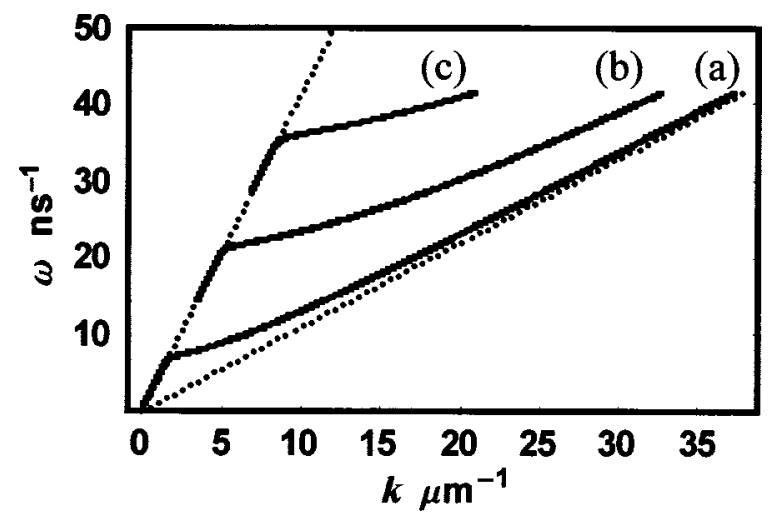

FIG. 2. Dispersion curves deduced from the Love mode phase speed curves in Fig. 1 using a $0.25 \mu \mathrm{m}$ thickness guiding layer; (a) first mode, (b) second mode, and (c) third mode. The upper dotted line corresponds to the substrate shear speed of $4160 \mathrm{~ms}^{-1}$ and the lower dotted line corresponds to the layer shear speed of $1100 \mathrm{~ms}^{-1}$.

thickness, $d$. Figure 2 shows the $(\omega, k)$ dispersion curve calculated from the solid curves Fig. 1 using $d=0.25 \mu \mathrm{m}$, so that $v_{l} / d=4.4 \times 10^{9} \mathrm{~s}^{-1}$. The solid curves show the first three Love wave modes, the upper dotted line corresponds to the substrate phase speed of $4160 \mathrm{~ms}^{-1}$, and the lower dotted line corresponds to the layer speed of $1100 \mathrm{~ms}^{-1}$. As anticipated from the form of Fig. 1, the $(\omega, k)$ dispersion curve for the first Love wave mode (curve a in Fig. 2) initially follows the relationship for a constant group speed equal to that of the substrate phase speed before deviating and joining the lower dotted line representing a constant group speed equal to that of the substrate phase speed. This pattern is repeated for the second (curve b in Fig. 2) and third (curve c in Fig. 2) Love wave modes, although a threshold frequency exists before each mode comes into existence. The slopes of the $(\omega, k)$ dispersion curves in Fig. 2 give the group velocities at any operating point. Figure 2 therefore shows that the group velocity for each of the Love wave modes goes through a minima at an intermediate value of $z$. The group velocities calculated from the slopes in Fig. 2 are shown as the dotted curves in Fig. 1. The group velocity is always less than the phase velocity and we therefore have a system with normal dispersion.

\section{Mass sensitivity}

An important factor in evaluating the potential usefulness of an acoustic wave sensor is the mass sensitivity, $S_{m}$, defined by the change in phase speed at fixed frequency, ${ }^{m}$

$$
S_{m}=\lim _{\Delta m \rightarrow 0} \frac{1}{\Delta m}\left(\frac{\Delta v}{v_{o}}\right)
$$

where $\Delta m$ is the deposited mass per unit area, and $v_{o}$ is the phase speed at the device operating frequency $f_{o}$; the mass sensitivity function is in units of $\mathrm{m}^{2} \mathrm{~kg}^{-1}$. For nonlayer guided acoustic plate mode (APM) devices, several authors have used the equivalent definition

$$
S_{m}=\frac{1}{v_{o}}\left(\frac{d v}{d m}\right)
$$

where $m$ is the mass per unit area on the APM device surface. Schumacher et al. ${ }^{18}$ commented that the mass sensitivity for an APM device, defined using frequency changes rather than phase speed changes, could be obtained by determining the slope of the curve at zero thickness. The relationship between the mass sensitivity and the slope of the phase speed with normalized mass layer thickness is evident from Eq. (2) by changing variables using $m=\rho_{l} d=\rho_{l} v_{l} z / f$ so that Eq. (2) becomes

$$
S_{m}=\frac{f}{\rho_{l} v_{l}}\left(\frac{d \log _{e} v}{d z}\right) \text {. }
$$

In the Love wave case, the deposited mass is in addition to the existing guiding layer and it is not immediately obvious that Eq. (3) continues to be valid. However, we have previously considered the problem of adding a perturbing mass layer to a Love wave device and shown that for a Love wave, $S_{m}$ can be rewritten in terms of the slope of the phase speed curve in Fig. 1 as, ${ }^{16}$

$$
S_{m}=\frac{1}{\rho_{l}}\left[\frac{1-v_{p}^{2} / v_{o}^{2}}{1-v_{l}^{2} / v_{o}^{2}}\right] \frac{f_{o}}{v_{l}}\left(\frac{d \log _{e} v}{d z}\right)_{z=z_{o}},
$$

where $v_{p}$ is the shear acoustic speed of the perturbing mass layer, $\rho_{l}$ is the density of the guiding layer, and $f_{o}$ is the operating frequency at the operating point $z_{0}$. The sensitivity formula, Eq. (4), for the Love wave device differs from the nonlayer guided formula, Eq. (3), only by a prefactor involving the shear acoustic speeds of the guiding layer and the perturbing mass layer. This prefactor is equal to unity when sensing a material with the same shear acoustic speed as the guiding layer and is approximately unity if both the layer and perturbing mass shear acoustic speeds are significantly less than the Love wave speed.

Figure 1 suggests that when the device operating point, $z_{o}$, is at the maximum sensitivity, the group and phase velocity will be significantly different. This implies that measurements of group and phase velocity may be used to deduce the mass sensitivity of a Love wave device. To consolidate this idea, we reconsider the definition of the group velocity and write it in terms of the $z$ parameter assuming a constant guiding layer thickness, $d$. The inverse group velocity is $v_{g}^{-1}=d k / d \omega$ and since $k=\omega / v$ we find

$$
\frac{v}{v_{g}}=1-\frac{\omega}{v} \frac{d v}{d \omega}
$$

and using $\omega=2 \pi z v_{l} / d$ gives

$$
\frac{v}{v_{g}}=1-z\left(\frac{d \log _{e} v}{d z}\right) \text {. }
$$

Using the approximation that $v_{p} \approx v_{l}$, we can then replace the term in the slope of $\log _{e} v$ in Eq. (4) by the mass sensitivity function, $S_{m}$, and obtain,

$$
\frac{v}{v_{g}} \approx 1-\rho_{l} d S_{m}=1+\rho_{l} d\left|S_{m}\right| .
$$

Since the sensitivity function, $S_{m}$, for the Love wave is negative, Eq. (7) predicts that the group velocity will always be less than the phase velocity. Equation (7) can also be 


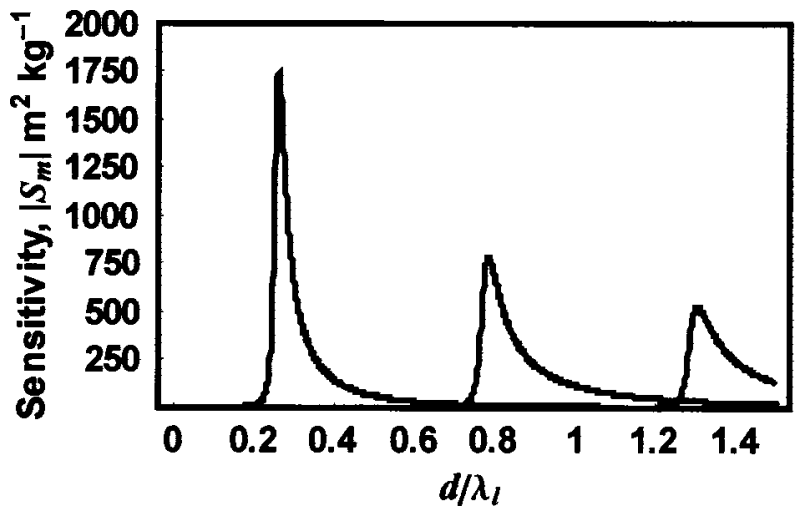

FIG. 3. Magnitude of mass sensitivity, $\left|S_{m}\right|$, evaluated from the group and phase velocities for the data in Fig. 1 using a frequency of $100 \mathrm{MHz}$.

rearranged to give the mass sensitivity as a function of the phase and group velocities and the guiding layer thickness and density,

$$
S_{m} \approx \frac{1}{\rho_{l} d}\left(1-\frac{v}{v_{g}}\right)=\frac{1}{\rho_{l} d} \frac{\left(v_{g}-v\right)}{v_{g}} .
$$

Thus, the mass sensitivity can be expressed as a fractional deviation of the phase velocity from the group velocity divided by the mass per unit area due to the guiding layer. Figure 3 shows the modulus of the mass sensitivity evaluated using Eq. (8) and the data for the three Love wave modes in Fig. 1; a frequency of $100 \mathrm{MHz}$ has been used in the calculation of Fig. 3.

From an experimental perspective, Eq. (8) is particularly important because it predicts that we should be able to evaluate the mass sensitivity of a Love wave device by making measurements of the group and phase velocities. We also believe that Eq. (8) can be applied to any nonlayer guided acoustic wave sensor, including APM, STW, shear horizontal SAW, and Rayleigh-SAW devices, simply by taking $\Delta m$ $=\rho_{l} d$ to be the deposited mass per unit area and examining the limit $\Delta m \rightarrow 0$. While Eq. (8) has been written as an approximate equality, for a non-Love wave sensor satisfying the mass sensitivity formula of Eq. (3), rather than Eq. (4), the equality will be exact.

An additional observation on the mass sensitivity is that the slope of the group velocity curve in Fig. 1 appears steeper than that of the phase speed. By analogy to Eqs. (3) and (4), which use the differential of $\log _{e} v$ with respect to the mass of the guiding layer, we can introduce a definition of the mass sensitivity based on the group velocity

$$
S_{m}^{g}=\left(\frac{d \log _{e} v_{g}}{d m_{l}}\right)_{d=d_{o}}=\frac{f_{o}}{\rho_{l} v_{l}}\left(\frac{d \log _{e} v_{g}}{d z}\right)_{z=z_{o}} .
$$

From comparing the curves in Fig. 1, we note that this group velocity mass sensitivity, $S_{m}^{g}$, may be larger than the mass sensitivity, $S_{m}$, defined using the phase velocity. For the data in Fig. 1, the peak in the group velocity mass sensitivity will also be sharp and then reduce to zero as the group velocity goes through its minimum. Subsequent to this minimum, the group velocity mass sensitivity will change sign as the group velocity approaches the value of the acoustic shear speed of

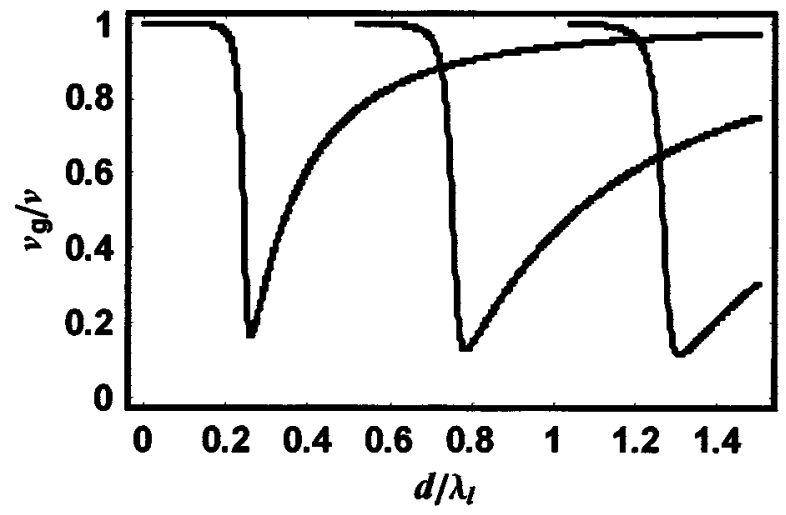

FIG. 4. The ratio of group to phase velocities evaluated for the data in Fig. 1 .

the layer. At the present time, it is not clear whether this group velocity mass sensitivity will be of value experimentally because the relative accuracy with which measurements of group and phase velocity can be made in Love wave sensors has not been widely investigated.

The definition of group velocity mass sensitivity used in Eq. (9) is similar, to within an overall negative sign, to the definition of phase velocity mass sensitivity given by Teston et al., in their work on the mass sensitivity of acoustic plate modes. ${ }^{10}$ They also note that a frequency mass sensitivity, $S_{m}^{f}$, can be defined in a similar manner to Eq. (3) and that it is related to the phase velocity mass sensitivity by $S_{m}^{f}$ $=S_{m} v_{g} / v$. This relationship has also previously been quoted by a number of authors in relation to work on APM sensors. Applying this formula to Eq. (8) gives

$$
S_{m}^{f}=\lim _{\Delta m \rightarrow 0} \frac{1}{\Delta m}\left(\frac{\Delta f}{f_{o}}\right) \approx \frac{1}{\rho_{l} d} \frac{\left(v_{g}-v\right)}{v} .
$$

Thus, the frequency mass sensitivity differs from the phase velocity mass sensitivity by whether the difference between group and phase velocities is expressed as a fraction with respect to the phase or group velocity. Since the phase velocity is always larger than the group velocity, $S_{m}^{f}$ will be smaller than $S_{m}$, possibly by an order of magnitude depending upon the operating point. To illustrate this point, Fig. 4 shows the ratio of group to phase velocity calculated for the data in Fig. 1. This difference between the types of mass sensitivity needs to be emphasized, because there is no difference between phase and frequency based mass sensitivity for a quartz crystal microbalance (QCM) when operated with no coating layer. Therefore, any relative comparison of a QCM to a Love wave device will depend on whether phase velocity or frequency based mass sensitivity is used.

\section{COMPARISON TO EXPERIMENT}

The Love wave system was created using a polymer guiding layer (PMMA from Aldrich) spin coated at $6000 \mathrm{rpm}$ across a SAW delay line device fabricated on $36^{\circ} X Y$ $\mathrm{LiTaO}_{3}$. The propagation direction was along the crystalline $x$ axis, which supports both a surface skimming bulk wave (SSBW) and a shear horizontal-SAW with speeds both approximately equal to $4160 \mathrm{~ms}^{-1}$. The polymer guiding layer 


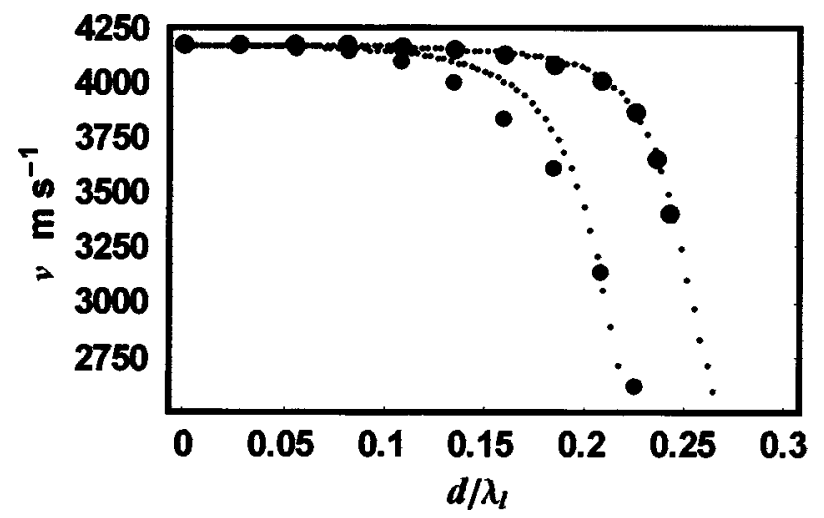

FIG. 5. Experimental data for variation of Love wave phase and group velocity with increasing guiding layer thickness (upper and lower data, respectively). The dotted curves are fits from Love wave theory.

covered the whole device, including the interdigital transducers (IDTs), and converts the acoustic modes into a Love wave. The IDT's consisted of a double-double split finger type design with a wavelength of $\lambda_{I D T}=45 \mu \mathrm{m}$. Each metal finger in the IDT was of width $6.75 \mu \mathrm{m}$ and each space between the fingers was $4.5 \mu \mathrm{m}$; the double-double design minimizes triple-transit interference. The uncoated SAW device had a resonant frequency of $92.64 \mathrm{MHz}$. Each IDT was of length $40 \lambda_{\text {IDT }}$ with an aperture of $65 \lambda_{\text {IDT }}$ and the separation between IDTs provided a center-to-center propagation path of $9.011 \mathrm{~mm}$. To obtain a range of guiding layer thicknesses, the polymer was successively spin coated across the whole device and then the device hardbaked at $200^{\circ} \mathrm{C}$ for 45 min. After each spin- coating step, the frequency spectrum of the device was measured and the resonant frequency and the corresponding group time delay recorded using a network analyzer (Agilent 8712ET). The phase velocity was deduced from the frequency change at minimum insertion loss and the group velocity from the group time delay at the frequency corresponding to minimum insertion loss; the error on the group velocity measurement was around $\pm 10 \%$. To provide an independent measurement of the group velocity, a separate pulse mode system was also used to measure the transit time of a short (100 ns) pulse of $\mathrm{rf}$ at the same resonant frequency and group velocities were subsequently calculated. The pulse mode system used for these experiments has already been described in detail in a previous report. ${ }^{13}$ The results showed that group velocities calculated from the two methods agreed to within 5\%.

The points in Fig. 5 show the measured phase and group velocities (upper and lower points, respectively) plotted as a function of $z=d / \lambda_{l}$, where $\lambda_{l}=v_{l} / f$. The dotted curves are fits of the Love wave theory to the data points, based upon an elastic mass guiding layer using $\rho_{l}=2600 \mathrm{kgm}^{-3}$ and $v_{l}=1100 \mathrm{~ms}^{-1}$. The value of $\rho_{l}$ used in fitting the Love wave theory to the data is significantly different from the measured value of $\rho_{l}=1100 \mathrm{~kg} \mathrm{~m}^{-3}$ and is needed to provide a less sharp and more rounded curve through the data points in the region $d / \lambda_{l} \sim 0.2$. We also performed experiments using Love waves generated from an SSBW mode on STQuartz and again needed to use an effective guiding layer density to accurately fit the data in the region $d / \lambda_{l} \sim 0.2$. The

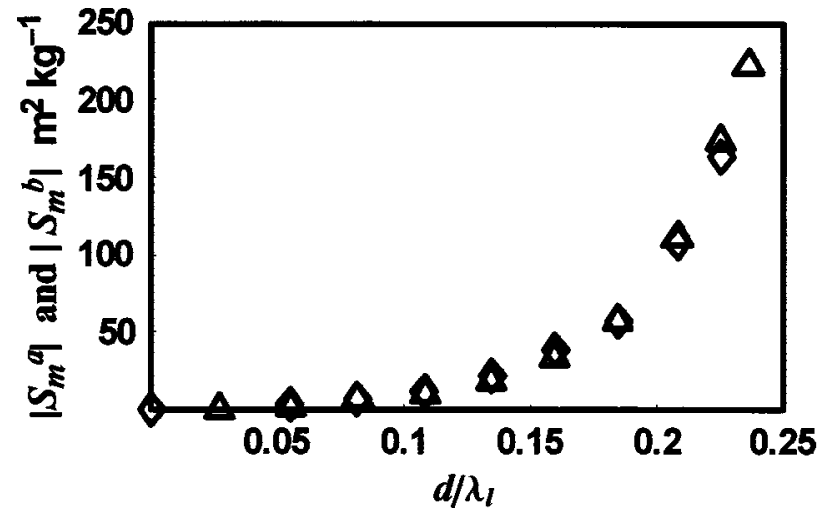

FIG. 6. Mass sensitivity with increasing guiding layer thickness: $\left|S_{m}^{a}\right|$ (triangles) by using phase velocity and Eq. (4), $\left|S_{m}^{b}\right|$ (diamonds) by using phase and group velocities and Eq. (8).

reasons for the need for an effective layer density are not obvious, but two possible candidates are the use of the SSBW mode to generate the Love wave and the use of a viscoelastic polymer guiding layer rather than an elastic solid. In either case, the precise fitting parameters used do not influence the comparison between the two methods of determining the experimental values of mass sensitivity, which is the purpose of these experiments. The layer shear speed used in fitting the data is consistent with values known for PMMA and is consistent with data for higher-order Love wave modes. ${ }^{19}$

To obtain two estimates of mass sensitivity, $S_{m}^{a}$ and $S_{m}^{b}$, from the experimental data we use Eqs. (4) and (8), respectively. Rewriting Eq. (4) using $x$ for the guiding layer thickness and using $v_{p}=v_{l}$ gives

$$
S_{m}^{a}=\frac{1}{\rho_{l}}\left(\frac{d \log _{e} v}{d x}\right)_{x=d} .
$$

The data for the phase speed was used to obtain simple estimates of the slope of $\log _{e} v$, using the difference between one value and the next and, hence $S_{m}^{a}$, at the experimental thicknesses. The second estimate of sensitivity, $S_{m}^{b}$, was obtained using Eq. (8). These two estimates are plotted against normalized thickness in Fig. 6; $\left|S_{m}^{a}\right|$ is indicated by triangles and $\left|S_{m}^{b}\right|$ by diamonds. It is notable that the absolute value for sensitivity is highly sensitive to changes in the estimate of the layer thickness, $d$. An error in calibration of the guiding layer thickness can have a significant effect on the absolute value of the sensitivity, while not altering the overall shape of the curve. While Eq. (11) has only one explicit factor of $d$, it should be noted that the phase speed is also dependent on $d$ through the combination of $d f / v_{l}$ and this increases the significance of any error in $d$. To further compare the two methods of estimating mass sensitivity, Fig. 7 plots $\left|S_{m}^{a}\right|$ against $\left|S_{m}^{b}\right|$. Although there is a slight offset of -2.6 on the intercept, the slope on this graph is 1.06 close to unity indicating the two methods are consistent. If $S_{m}^{a}$ is calculated from the experimental data using backward differences of the slope of $\log _{e} v$, slightly different values of slope and intercept occur, but the slope remains close to unity. 


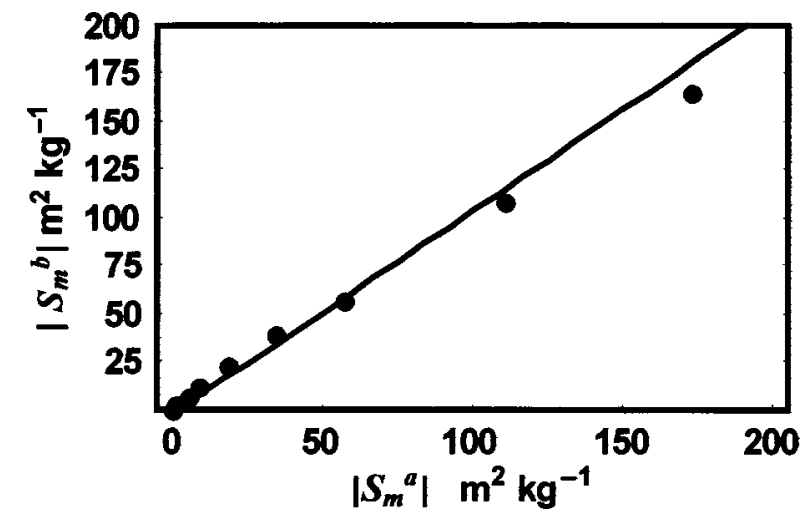

FIG. 7. Comparison of the two methods of measuring mass sensitivity; solid line is a best fit with a slope of 1.06 .

Figure 1 indicates that the change in group velocity with guiding layer thickness is more rapid than that of the phase velocity. The experimental data in Fig. 5 confirm this for the all values of $z$ at which direct comparisons can be made. The slope of the phase velocity curve at the two highest values of $z$ may be less accurate because this corresponds to a large insertion loss. The data therefore confirms the idea that the group velocity is a sensitive parameter to mass deposition. Rewriting Eq. (9) in a form similar to Eq. (11) gives,

$$
S_{m}^{g}=\frac{1}{\rho_{l}}\left(\frac{d \log _{e} v_{g}}{d x}\right)_{x=d},
$$

and $S_{m}^{g}$ can be evaluated from the experimental data in a manner similar to the calculation of $S_{m}^{a}$. In Fig. 8, both forward and backward differences have been averaged to provide the data points. The group velocity based mass sensitivity provides a significant enhancement over the phase velocity mass sensitivity at low to moderate guiding layer thicknesses, although further work is needed to determine the relative accuracy with which the two types of mass sensitivity can be determined. In this work, we have used a network analyzer to determine phase and group velocity, with a separate pulse mode system to confirm the group velocity measurements. However, practical sensors are often based on simple circuits implementing the measurement of phase velocity via phase shifts typically using a mixing between a detected signal and a reference continuous wave. This type

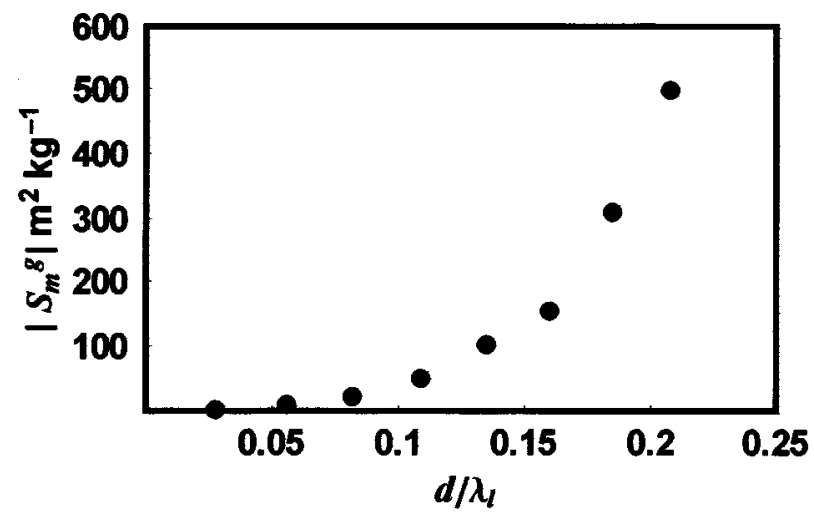

FIG. 8. Sensitivity defined using the slope of the group velocity [Eq. (12)]. of phase measurement achieves a significant accuracy because the measurement is resolved to within a few percent of the wavelength; achieving the same accuracy with a group velocity based sensor system is more difficult. However, the significant enhancement achievable via the group velocity over the phase velocity mass sensitivity at low to moderate guiding layer thicknesses (for a given Love wave mode) may prove equally important as this is the range of guiding layer thickness to which the sensor is likely to be limited for the first Love wave mode given the high insertion loss with large polymer thickness.

\section{CONCLUSION}

It has been shown that dispersion in acoustic wave sensors is an intrinsic and important property when they are used for mass sensing. This dispersion is particularly strong in Love wave devices and is strongly related to the high mass sensitivity that these sensors possess. A formula relating the difference in group and phase velocities to the mass sensitivity has been derived and this formula is applicable to both Love wave and other acoustic wave sensors. The applicability of this formula has been confirmed experimentally using a Love wave device consisting of a polymer on an $36^{\circ} X Y$ Lithium Tantalate substrate operated at a frequency around $93 \mathrm{MHz}$. It has also been suggested that the group velocity may be a good sensor parameter.

\section{ACKNOWLEDGMENT}

Two of the authors (G.M. and M.I.N.) gratefully acknowledge the BBSRC for financial support under research Grant No. 301/E11140.

${ }^{1}$ M. J. Vellekoop, Ultrasonics 36, 7 (1998).

${ }^{2}$ E. Gizeli, M. Liley, C. R. Lowe, and H. Vogel, Anal. Chem. 69, 4808 (1997).

${ }^{3}$ I. D. Avramov, S. Kurosawa, M. Rapp, P. Krawczak, and E. I. Radeva, IEEE Trans. Microwave Theory Tech. 49, 827 (2001).

${ }^{4}$ A. J. Ricco and S. J. Martin, Appl. Phys. Lett. 50, 1474 (1987).

${ }^{5}$ E. Gizeli, A. C. Stevenson, N. J. Goddard, and C. R. Lowe, IEEE Trans. Ultrason. Ferroelectr. Freq. Control 39, 657 (1992).

${ }^{6}$ G. Kovacs and A. Venema, Appl. Phys. Lett. 61, 639 (1992).

${ }^{7}$ M. Rapp et al., IEEE Ultrasonics Symposium Proceedings, edited by M. Levy, S.C. Schneider, and B.R. McAvoy, 1995, Vols. 1 and 2.

${ }^{8}$ R. M. White, P. J. Wicher, S. M. Wenzel, and E. T. Zellers, Freq. Control UUFFC-34, 172 (1987)

${ }^{9}$ S. Liew et al., IEEE Ultrasonics Symposium Proceedings, edited by B.R. McAvoy, 1990, Vols. 1-3.

${ }^{10} \mathrm{~F}$. Teston, G. Feiullard, L. Tessier, and M. Lethiecq, IEEE Trans. Ultrason. Ferroelectr. Freq. Control 45, 2666 (1996).

${ }^{11}$ C. McMullan, H. Mehra, E. Gizeli, and C. R. Lowe, J. Phys. D 33, 3053 (2000).

${ }^{12}$ F. Herrmann and M. Weihnacht, Proc.-IEEE Ultrason. Symp. 1, 413 (1999).

${ }^{13}$ M. I. Newton, G. McHale, F. Martin, E. Gizeli, and K. Melzak, Analyst (Cambridge, U.K.) 126, 2107 (2001).

${ }^{14}$ A. C. Stevenson, H. M. Mehta, R. S. Sethi, L-E. Cheran, M. Thompson, I. Davies, and C. R. Lowe, Analyst (Cambridge, U.K.) 126, 1619 (2001).

${ }^{15}$ G. McHale, M. I. Newton, and F. Martin, J. Appl. Phys. 91, 5735 (2002).

${ }^{16}$ G. McHale, M. I. Newton, and F. Martin, J. Appl. Phys. 91, 9701 (2002).

${ }^{17}$ D. S. Ballantine, R. M. White, S. J. Martin, A. J. Ricco, E. T. Zellers, G. C. Frye, and H. Wohltjen, Acoustic Wave Sensors (Academic, New York, 1997).

${ }^{18}$ J. Schumacher, R. Dahint, F. Josse, and M. Grunze, Proc.-IEEE Ultrason. Symp. 1, 629 (1994).

${ }^{19}$ G. McHale, M. I. Newton, F. Martin, E. Gizeli, and K. A. Melzak, Appl. Phys. Lett. 79, 3542 (2001). 